\title{
Socioeconomic and Cultural Constraints in the Management of Bladder Rhabdomyosarcoma in a Resource Poor Setting
}

\author{
Okoro Jude Chidi ${ }^{1}$, Emechebe George ${ }^{1}$, Ezeogu Joseph ${ }^{1}$, Onyenwe Nathaniel Ejikeme ${ }^{2}$ \\ ${ }^{1}$ Department of Pediatrics, Faculty of Medical Sciences, Imo State University, Orlu, Nigeria; ${ }^{2}$ Department of Pharmaceutical \\ Microbiology, Prof. Dora Akunyili College of Pharmacy, Igbinedione University Okada, Edo State, Nigeria.
}

\section{Corresponding Author: \\ Dr Okoro Jude Chidi \\ Email: jubhildr@yahoo.com}

This is an Open Access article distributed under the terms of the Creative Commons Attribution License (creativecommons.org/ licenses/by/3.0).

Received : December 13,2018

Accepted : May 8, 2019

Published : May 25, 2019

\begin{abstract}
Background: With improvement in the control of infection and malnutrition, noncommunicable diseases such as cancer may account for significant proportion of morbidity and mortality amongst under five children in future. We are hoping through this paper to drive home the reality and extent of the problem that patients, families, healthcare providers are confronted within the management of children with cancer. Case Report: A 3 year old male from poor socio-economic status presented with inability to pass urine, abdominal mass, generalized body swelling, fecal incontinence and rectal prolapse. Abdominopelvic CT scan, cystoscopy revealed a bladder mass involving both ureters, hydronephrosis and pelvicalyceal dilatation. Histological report showed sheets of rounded and spindle cells in a myxoid stroma with tumor cells showing visible cross striations, suggesting diagnosis of bladder rhabdomyosarcoma. Patient was jointly managed with the paediatric surgical team. Conclusion: Late presentation, ignorance, poverty, lack of appropriate equipments and manpower were notable constrains against effective management of this patient with bladder rhabdomyosarcoma.
\end{abstract}

Keywords: Child, Hydronephrosis, Nigeria, Rhabdomyosarcoma, Ureter, Urinary Bladder.

\section{Introduction}

Rhabdomyosarcoma (RMS) is the commonest soft tissue sarcoma (STS) in childhood constituting about $40 \%$ of all soft tissue sarcoma and $5 \%$ of all childhood tumors [1]. Majority are sporadic and occur before the age of 5 years [1,2]. Brown and Oluwasola in Ibadan South West of Nigeria in their study on childhood rhabdomyosarcoma revealed that embryonic type is the commonest variant $(61 \%)$, while the commonest site is head and neck (51\%) [3]. Occurrence of the tumor in the genitor-urinary tract may present with hematuria, obstruction of the lower urinary tract, recurrent urinary tract infection, incontinence, or a mass detectable by abdominal or rectal examination [4]. Poverty and low educational achievement being a correlate of lower socioeconomic status have significant impact on the management of these patients leading to ultimately poor outcome

\section{Case Report}

The patient is a 3 year old male leaving in the rural area of South East Nigeria who presented with inability to pass urine, abdominal mass and generalized body swelling. The problem started six months prior to presentation with initial increase in frequency of urination, pain on micturition, straining on micturition and dribbling. Three weeks prior to presentation, he developed generalized body swelling, rectal prolapse and fecal incontinence. Inability to pass urine developed two days before presentation to our hospital. In the course of illness, his parents took him to see a laboratory technologist who prescribed some medication 
following a laboratory test; a traditional doctor, where he received herbal medication; and the church to receive healing. During this period the child's clinical condition continued to deteriorate. The father is a menial worker while the mother is a petty trader; both parents have primary education only.

Examination at presentation revealed a chronically ill looking child in painful distress, moderately pale, febrile, generalized edema, distended urinary bladder, ballotable tender kidney, normal external genitalia and suprapubic mass. A clinical diagnosis of bladder outlet obstruction secondary to a pelvic tumor was made. Posterior urethral valve and urethral stricture were considered as differentials. Full blood counts, urine analysis, urine microscopy and culture showed nomocytic hypochromic anemia, severe urinary tract infection due to Pseudomonas aerogenosa. Serum electrolytes were normal while serum urea and creatinine levels were elevated with a calculated GFR of $58 \mathrm{ml} / \mathrm{min} / 1.73 \mathrm{~m}^{2}$. Abdominopelvic scan revealed an echogenic mass with irregular outline in the posterior part of the bladder and severe kidney pelvicalyceal dilatation. However the cortico-medullary differentiation was preserved. The abdomino-pelvic CT scan showed an enhancing heterogenous urinary bladder mass with bilateral moderate hydronephrosis and hydroureter. Cystoscopy revealed a soft grey grape like rounded mass involving the opening of the ureters. Histological report of biopsy done during cystoscopy showed sheets of rounded and spindle cells in a myxoid stroma. Tumor cells showed visible cross striations. A diagnosis of rhabdomyosarcoma of the bladder was made and the pediatric surgical unit was invited to review the patient. Urethral catheter was inserted for bladder drainage. The urethral catheters were repeatedly getting blocked necessitating periodic examination and changes.

Antibiotics were prescribed to treat urinary tract infections. Unfortunately on several occasions antibiotics prescribed were not available resulting in poor compliance to drug therapy. Non-availability of funds was cited as the reason for not providing the antibiotics though the spectrum of antibiotics in the hospital pharmacy was very narrow. There was no government or hospital policy recognizing indigent patients. Most of the fund used in buying drugs and paying for the tests were contributions from attending doctors, medical students and well meaning individuals. The patient was initially booked for surgery to relieve obstruction; however this was revised following cystoscopic findings. A large tumor was observed involving most of the bladder wall and obliterating the orifice of both urethras. Chemotherapy and radiotherapy was then advised to reduce the tumor bulk. Radiotherapy was not available and chemotherapy was never commenced until the demise of the child. Actinomycin D was not available locally and lack of fund was a huge impediment in procuring the drugs. During the course of the illness child became severely anemic necessitating blood transfusions to correct anemia and hematological abnormalities prior to commencement of chemotherapy. Renal function continued to deteriorate mainly because of the inability of the pediatric surgeons to relieve the ureteric obstruction due to non availability of ureteric stent, and reluctance of the pediatric surgeons in caring out a nephrostomy resulting in progressively worsening bilateral hydronephrosis and renal failure.

\section{Discussion}

Our patient presented late to hospital, six months after the onset of illness. During this period, he was taken to a medical laboratory technologist, a traditional doctors, patent medicine dealers and spiritual houses for treatment. Inevitably, the disease condition worsened preventing timely and adequate treatment. Ineffective treatment received from these quacks may have complicated the disease process. It is not surprising that our patient ended up with very poor outcome. Gitau J et al. [5] in their work attested to the fact that poor outcome 
has been associated with late presentation and is a major contributor to under five mortality rate. Late presentation to hospital is a common phenomenon in this part of the world as reported by many workers [6-8]. Anolue and co workers [9] defined late presentation as presentation to hospital three months after the onset of symptoms. Common reasons adduced for late presentation in this part of Nigeria will include exploring other treatment options at home such as herbal medication and procuring drugs from patent medicine dealers, living far from hospital and low socioeconomic status [8]. Issues bordering on caregivers perception of the origin of diseases has been raised especially by some authors [10]. Most of our patients believe that diseases are caused by evil spirits, This is a reflection of deep seated cultural beliefs [11]

Drugs such as antibiotics, chemotherapeutic agents and essential laboratory tests required to manage the patient could not be provided by the parents due to paucity of funds. This is in the light of the fact that a significant number of children presenting to children emergency room in this part of the world have low socioeconomic status with most of the parents by WHO definition of poverty earning below the poverty line. Several studies in this part of Nigeria shows that a significant number of children with low socioeconomic status presenting to the hospitals have poor outcome similar to our index case $[12,13]$. However it is commendable that some tertiary institutions especially Federal institutions have policies to identify indigent patients, with a view of supporting these patients to obtain most effective treatment available. Some non-government organization provides facilities for free or subsidized treatment for certain categories of diseases such as tuberculosis, leprosy and acquired immune deficiency syndrome in children; unfortunately, childhood tumors are not included. IMSUTH as a tertiary institution unfortunately does not have any of these policies on ground. This is rather unfortunate because serious ethical issues bordering on neglect of patients may be raised against the attending medical doctors in the course of managing this child. Poor educational attainment and poverty of the parents of our patients was noted to be significant constraints to effective management of the patient. Parental ignorance stemmed from poor educational attainment resulting in inappropriate medical attention for their child led to late presentation. Obi-Nwosu et al. [14] working in the South Eastern part of Nigeria showed clearly that educational status of the caregiver significantly affected their health seeking behavior. Demand for healthcare facilities is a function of the level of education because they are more enlightened about orthodox medicine over traditional health care.

IMSUTH is a state owned tertiary institution, it is providing medical education and training to health professional and also serving as a research centre. In line with this, it should have appropriate medical equipment to aid in diagnosis, monitoring or treatment of medical conditions. In the management of our patient, the hospital lacked both facilities and manpower to effectively manage this patient. Most of the investigations such as serum electrolytes, urea and creatinine, CT scan and histology report on the biopsies were done in laboratories outside the hospital resulting in increasing cost, delaying diagnosis and commencement of treatment. Oladejo et al. [15] working in South Eastern part of Nigeria reported in their study that most tertiary hospital facilities in Southeast Nigeria are poorly equipped with poor maintenance culture. The need for effective and adequate support facilities such as laboratory services within the institution and the dangers of patronizing laboratory facilities which are not under the supervision of the institution cannot be over emphasized. This may raise serious issues of ethical consideration and quality of services rendered at these laboratories.

The nature and location of the tumor posed a great challenge to early diagnosis and 
commencement of treatment; it also contributed to late presentation to hospital. The location of a tumor determines the ease to which it can be assessed, which invariably affects early diagnosis and effective management. The impact of tumor location on the prognosis of patient has been a subject of series of research. Recently, a retrospective study [16] revealed that ureteral tumors had a worse prognosis. The diagnosis and effective management of these children required specialized equipments and highly skilled personnel for highly specialized procedures such as nephrostomy and radical nephro-uretectomy that are not available in the hospital.

\section{Conclusion}

Most of the state owned tertiary health institutions are unprepared to play key roles in the management of these children as shown by our index case. It is also important to recognize the socio-demographic characteristics of these children as exemplified by our index case. These include low socioeconomic status (poverty and poor educational attainment of the parents), delayed presentation to hospital, ill equipped hospital with lack of specialized manpower, lack of appropriate hospital and government health policies to effectively manage these indigent children.

Contributors: OJC: manuscript writing, patient management; EG. EJ: manuscript editing, patient management; ONE: critical inputs into the manuscript and patient management. OJC will act as guarantor. All authors approved the final version of this manuscript.

Funding: None; Competing interests: None stated.

\section{References}

1. Stiller CA, Parkint DM. International variations in incidence of childhood soft tissue sarcomas. Paediatric and Perinatal Epidemiology. 1994;8(1):1365-3016.

2. Ognganovic S, Linabery AM, Charbonneau B, Ross JA. Trends in childhood rhabdomyosarcoma incidences and survival in the United States (1975-2005). Cancer. 2009;115(18):4218-4226.

3. Adigun IA, Rahman GA, Buhari MO, Ogundipe KO, Omotayo JA. Pattern of rhabdomyosarcoma in Nigeria children. J Natl Med Assoc. 2008;100:906-909.
4. Babatunde TO, Akang BJ. Pattern of childhood cancer in university college hospital ugandan during 1991-2010 and comparison with the previous three decades. Paediatric and International Child Health. 2015;35(2):144.

5. Gitau J, Irimu G, Wamalwa D, Njai D. Factors associated with late presentation to Kenyatta national hospital for acute severe illness in children aged 2-59 months. Pinnacle Medicine and Medical Sciences. 2016;3(2).

6. Oladeji A, Alalabi O, Jimoh M, Ntekim I, T Elumelu T. Delay in presentation of cancer patients for diagnosis and management: An institutional report. The Internet Journal of Oncology. 2016;13:1.

7. Adisa AO, Arowolo OA, Akinkuolie AA, Titiloye NA, Alatise Ol, Lawal OO, et al. Metastatic breast cancer in Nigerian tertiary hospital. Afr Health Sci. 2011;11(2):279-284.

8. Adesina OA, Olasoji OH, Ugboko VIO. Reasons for late presentation of cleft deformity in north eastern Nigeria. Kanem J of Med Sci. 2010;4(1):25-27.

9. Anolu RI, Orakwele CO, Oyeneyiu L, Abudu OO. Late presentation of patients with cervical cancer to a tertiary hospital in lagos. What is responsible? Eur J Gynaecoloncol. 2004;25(6):729-732.

10. Meremikwu MM, Elurie JE, Nkanga DG, Udoh EE, Ikpatt OF, Olaje EO. Socioeconomic constraints to effective management of Burkitt's lymphoma in southeastern Nigeria. Tropical Medicine and International Health. 2005;10:92-98.

11. Abubakar A, Van B, Fisher R, Bomu G, Gona JK, Newton CR. Sociocultural determinants of health seeking behavior on Kenyan coast. A qualitative study. Plos ONE. 2013;8(11):e71998.

12. Adegboye MA. Socioeconomic status categories of rural dwellers in northern Nigeria. Advances in Research. 2016;7(2):1-10.

13. Olaogun AAE, Adebayo AA, Ayandiran OE, Olasode OA. Effects of mother's socioeconomic status on the management of febrile conditions in under five children in a resource limited setting. BMC International Health and Humans Rights. 2006:6(1):1-6.

14. Obi-Nwosu AL, Nwosu OB, Nnaji G. Family and social determinants of health-seeking behaviour of caregiver of Febrile children in an urban city of South Eastern Nigeria. Arch Med. 2016;8:3.

15. Oladejo EJ, Umeh OC, Egolum CC. The challenges of health care facilities maintenance in tertiary hospital in south eastern nigeria. International J of Civil Engineering Construction and Estate Management. 2015;3:1-6.

16. Wu YJ, Dong Q, Liu L, Han P, Wei Q. The impact of tumour location and multi focality on prognosis for patients with upper tract urothelial carcinoma. A metaanalysis. Sci Rep. 2014;4:6361. 